\title{
Teleplanning by Human Demonstration for VR-based Teleoperation of a Mobile Robotic Assistant
}

\author{
Costas S. Tzafestas \\ Institute of Informatics and Telecommunications \\ National Center for Scientific Research "Demokritos", \\ 15310 Aghia Paraskevi, Athens, Greece \\ E-mail: ktzaf@iit.demokritos.gr
}

\begin{abstract}
This paper focuses on the integration of local path planning techniques in a multimodal teleoperation interface, for the efficient remote control of a mobile robotic assistant. The main principle underlying this scheme is related to the continuous efforts in finding new ways to establish an efficient human-robot cooperation framework, where humans and robots take in charge the parts of the tasks that they can perform more efficiently. For the teleoperation of a mobile robotic platform, a simple application of this general principle could be to commit the human operator in performing the necessary global planning operations, which are more demanding in terms of complex reasoning and required "intelligence", while other more local tasks such as collision avoidance and trajectory optimization are dedicated to the telerobotic system. We propose an implementation of this principle in a mobile robot teleoperation interface integrating virtual reality techniques and Web-based capabilities. This paper describes the multimodal interface and the design principles followed, as well as the integration of a local path planning method. The method is based on wavefront expansion of a simple distance metric and the use of a trajectory modification technique similar to vector field approaches. This scheme, called "computer-assisted teleplanning by human demonstration", aims at providing active assistance to the human operator, enabling him to indicate in a natural way the desired global motion plan, for a more efficient teleoperation of a mobile robotic assistant.
\end{abstract}

Keywords - Telerobotics, virtual reality, human/robot cooperation, mobile service robots.

\section{INTRODUCTION}

During the last five to ten years, robot teleoperation technology has been constantly evolving to incorporate new technological advances in many fields, including: (i) robot sensors and actuators, with the development of novel sensor-based control

This research work was supported by the Greek General Secretariat for Research and Technology and the European Union, under grant PENE $\Delta-99-\mathrm{E} \Delta 623$ strategies, (ii) human-machine interaction, with the development of advanced multimedia interfaces including virtual reality (VR) techniques [7][9], as well as (iii) computer networks and communications, with the Internet being a typical example [8][11]. Telerobotics is a multidisciplinary research field intersecting many scientific domains, such as those mentioned above, and having as a primary goal to establish an efficient communication and cooperation framework between humans and robots. The human operator and the telerobotic devices must be enabled to work together and collaborate efficiently towards performing the desired physical tasks. To achieve such a "synergy" between the human operator and the robot, the teleoperation (master or slave) control system must support the following functionalities:

- Understand the "intentions" of the human operator and interpret his actions correctly. The human operator must be able to "indicate" in a natural manner the action plan that is to be followed in order to perform the desired physical task.

- Provide active assistance to the human operator, correcting his actions when necessary in order to deduce an appropriate action plan in the form of robot commands that have to be sent for execution to the slave robot controller.

- Supply "rich" multi-sensory feedback to the human operator, to help him interpret intuitively the state of the remote task and the actual or expected results of current or planned actions.

We thus see that in order to enable such an intuitive human-robot interaction and cooperation, the use of multimodal teleoperation interfaces, exploiting multisensory displays and VR technologies, is of primary importance. Moreover, some "intelligence" is required for the interface to interpret correctly human intentions and deduce appropriate 
robot action plans. The basic principle of such a collaborative teleoperation framework is to enable humans and robots to be in charge of the tasks and sub-tasks that they can accomplish more efficiently, depending on the specific situation and problem at hand and the related constraints. A simple application of this general principle for the teleoperation of a mobile robotic platform, could be to commit the human operator in performing the necessary global planning operations, which are more demanding in terms of complex reasoning and required "intelligence", while other more local tasks such as collision avoidance and trajectory optimization can be dedicated to the telerobotic system.

This paper proposes an implementation of this principle and its integration in a multimodal user interface for the teleoperation of a mobile robotic platform. We start by describing in Section II the teleoperation interface, the design principles followed and their implementation. The system integrates VR techniques and Web standards to facilitate teleoperation through the Internet, and increase interactivity as well as intuitive operation of the system. Section III describes the integration in the teleoperation interface of a local path planning method, which is based on wavefront expansion of a simple distance metric and the use of a trajectory modification technique similar to vector field approaches. The method aims at providing active assistance to the human operator, enabling him to indicate in a natural way the desired global plan to be followed by a remote mobile robot. This scheme, called "computer-assisted teleplanning by human demonstration", constitutes the first mode of teleoperation supported by the system, aiming at a more efficient and intuitive "teleprogramming" of our mobile robotic assistant. This robotic assistant consists of a mobile platform equipped with a variety of on-board sensing and processing equipment, as well as a small manipulator for performing simple fetch-and-carry operations. The work is performed in the framework of a research project, which has as a final goal the development of a mobile robotic system to perform assistive tasks in a hospital environment.

\section{Multimodal Teleoperation Interface}

\section{A. Design Principles}

In this section, we discuss and analyze some general guidelines and principles that need to be taken into account for the design of an efficient robot teleoperation system. Efficiency in remote operation and control of a robotic system concerns: (a) making "good use" of the available communication bandwidth between the master and slave systems, and (b) achieving a "synergy" between the human operator and the robot, by enabling the system to best exploit and integrate (in terms of speed, precision and error recovery) both: (i) the human operator capacity to take rapid decisions and intuitively indicate the most appropriate (coarse or detailed) plan for system action (e.g. robot motion) in complex situations, and (ii) the robotic system capacity to perform, with controlled speed and precision, a variety of autonomous (sensor-based) physical tasks.

To approach towards these general targets, a set of requirements have to be specified and fulfilled by the teleoperation system and all its submodules. The final system design must converge towards the merging between a number of often contradictory modalities, in search of an "optimum" compromise and increased "efficiency". By multimodal teleoperation interface we mean a system that supports: (a) multiple computer-mediated human/robot interaction media, including VR models and tools, or even natural gesture recognition etc., and (b) multiple modes of operation, with a varying degree of robot autonomy and, respectively, human intervention. The latter is a very important issue for the design of a telerobotic system. The modes of operation that we are considering and will be supported by the teleoperation system include the following (see [15][10] for a comprehensive survey on the evolution of teleoperation systems):

(a) Direct teleoperation control, based on an online master-slave exchange of low-level commands (e.g. move forward distance $d$ with speed $v$, rotate right $10^{\circ}$ etc.) and raw sensory feedback (velocity signal from the odometry, visual feedback from an on-board camera etc).

(b) Computer-aided master control of the mobile robot platform, with the computer system at the master control station providing some form of assistance to the human operator, such as: (i) performing information feedback enhancement, like for instance model-based predictive display, (ii) undertaking active control for some of the dofs of the system, (e.g by constraining the motion of the platform on a set of prespecified paths, related to the desired task), thus substituting or complementing some of the human operator's actions, or even (iii) provid- 
ing some form of active guidance to the human operator's actions, based on a VR model of the slave robot environment and a set of desired task models. In other words, this mode of teleoperation control is based on a set of functions supported by the master control system by performing active monitoring and real-time model-based correction of the human operator actions, to satisfy a number of task-related constraints. Two main functions are being initially integrated in our system: an active anti-collision and an active motion-guide function, both based on the use of either a virtual reality model of the robotic platform and its task environment, or of a simple 2D top-view representation.

(c) Shared-autonomy teleoperation control of the robotic system, using a set of sensor-based autonomous behaviors of the robot, such as a real-time automatic collision avoidance behavior, based on data from ultrasonic (or/and infrared) sensors. This mode of teleoperation control can be extended to incorporate a large set of intermediate-level, behavior-based, hybrid (qualitative/quantitative) instructions, such as for instance: move through point $A, B, C$ while avoiding obstacles, pass through the door on the left, move at distance $d$ from the wall on the right, follow corridor etc. These commands will trigger and make use of respective behavior-based control modes of the robot, incorporating automatic path generation functions. In other words, this mode of teleoperation control is based on some form of basic autonomy (local path planning and reactive sensorbased behaviors etc.) embedded on the slave robot. Of course, the master control system should enable this form of intermediate-level, behavior-based remote control by allowing the human operator to intuitively indicate the robot plan, interpreting his actions/indications and transforming them into appropriate robot instructions that fall into this category.

(d) Semi-autonomous teleoperation, based on a set of high-level qualitative task-based instructions, such as: go to location $X$, grasp object $A$ on table $B$ of room $C$ etc. This set of instructions must be built upon a combination of task-planning, path-generation and environment-perception modules that will be incorporated on the robot control system.

(e) High-level supervisory control, that is, simple monitoring of sensory feedback, and limited human intervention on specific complex situations, requir-
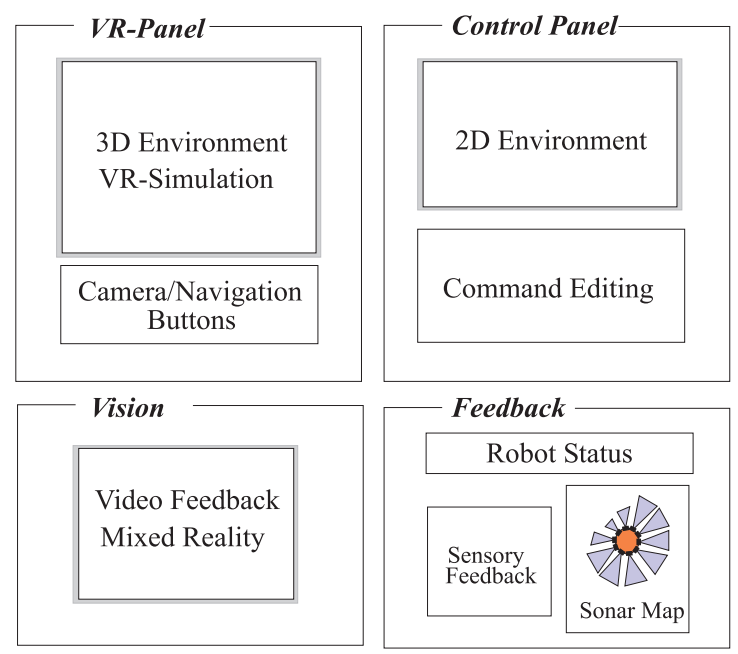

Fig. 1. Teleoperation interface: General layout

ing difficult decision making and task planning.

All these modes of teleoperation can be used for on-line monitoring and remote control of a mobile robot. The system, however, should also support some or all of these control modes in an off-line teleprogramming scheme, where the human operator controls the robot task in a simulated environment and checks the validity of his actions before actually sending the commands (registered action plan) to the slave robotic system for real execution. A combination of these control modes have to be considered for the teleoperation system of our mobile robotic assistant. Depending on the specific application and the tasks to be performed, only some of these modes, however, will become active at each time-instant.

\section{B. Implementation: VR-based Teleoperation}

The human/computer interface for the teleoperation of the mobile robotic assistant has the general layout shown in figure 1 . It consists of four main components:

(i) The VR-panel, where the 3D graphical models of the robotic system and its task environment are rendered. This simulation environment constitutes the first modality for inserting instructions (motion commands etc.) to the system in a natural and intuitive way. The human operator navigates within this virtual world and guides the virtual robot directly towards the desired target location. The input devices that will be used in the first place are: a joystick for virtual robot motion control, and a 
trackball for virtual camera control and navigation. The active assistance modules will have as a function to reduce the workload of the human operator by performing on-line motion/action correction according to task-related constraints. Moreover, some form of sensory feedback information is integrated in this virtual environment, like the actual robot position represented by a wireframe graphical model of the robot platform.

(ii) The control-panel, containing a 2D top-view graphical representation of the mobile robot environment (corridors, doors, rooms, obstacles etc.) and a command editing panel. The 2D graphical environment contains accurate map information of the whole indoor environment, where the robotic assistant will operate, allowing the human operator to obtain rapidly a top-view of any required region (using scrollbars or predefined region-buttons). The human operator will also have the ability, if needed, to directly edit commands that must be sent to the robot.

(iii) The sensory-feedback panel, where information on the actual status of the robot, as well as other required sensory feedback signals (except real video feedback) will be represented (for instance a sonar map, representing the location of obstacles detected by the robot).

(iv) The visual-feedback panel, where the images obtained by the on-board robot camera will be displayed. The refresh-rate of this video feedback will of course be reduced, since the bandwidth available for communication through the Internet is limited and real-time access to other more critical information, such as actual robot location and sensor status, is indispensable.

The human operator interface is developed based on Java technology, in order to facilitate Web-based operation of the system. Figure 2 shows a snapshot of the first-prototype version of this interface. We may notice: (a) the VR panel, which includes real-time animation of $3 \mathrm{D}$ graphical models for the mobile robotic platform and its task environment (corridors, rooms, doors etc.) (b) the $2 \mathrm{D}$ control panel, providing command editing functionalities, and (c) the feedback panel, supplying robot status information. The 3D graphics rendering routines for the VR panel are implemented using the Java3D API. An extended version of this human/computer interface will thus constitute in the near future the Internet-based teleoperation control platform for the mobile robotic assistant. Some development

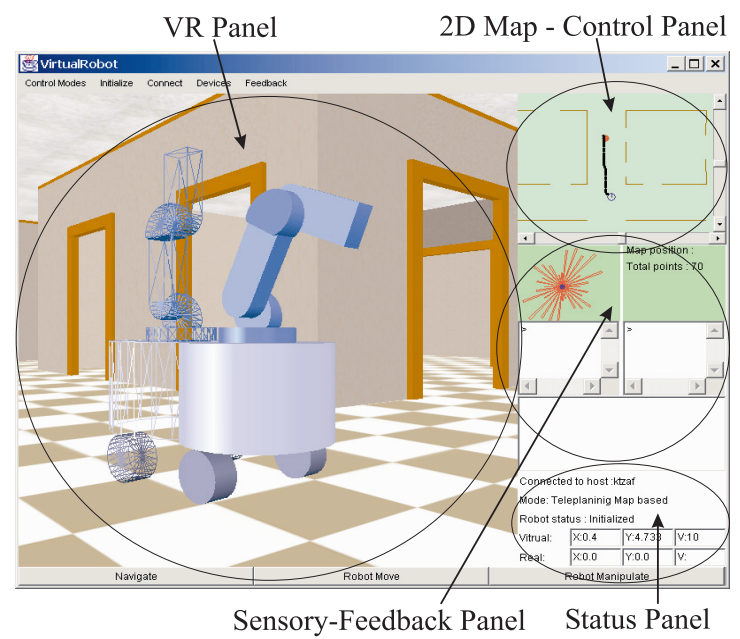

Fig. 2. Multimodal teleoperation interface for the mobile robotic assistant: First prototype implementation

that remains to be done includes a visual feedback panel, displaying real video images captured by the on-board robot camera.

\section{Mobile Robot Teleplanning By HUMAN DEMONSTRATION}

Off-line teleprogramming is the first control scheme to be implemented on the real telerobotic system, since it constitutes the safest mode of operation in the presence of large and variable time delays. The main goal is to automatically generate a correct robot action plan from observation of the actions performed by the human operator within the VR or the $2 \mathrm{D}$ control panel. A solution consists of registering critical waypoints containing information such as robot position and orientation, navigation speed, acceleration etc. Interpolation between these waypoints by the local navigation module of the robot must result in the desired motion for the mobile platform. Motion commands sent to the robot will thus contain a list of such waypoints with potential additional task-related information.

According to the general teleoperation design guidelines and objectives, outlined in the previous sections, the human operator should be able to indicate in a natural and intuitive way the desired task plan, while the system should be "clever" enough to understand his intentions, interpret his actions and correct them appropriately to deduce a suitable robot action plan, in the form of a series of robot commands (robot program). In this section we propose a method for providing active assistance to the 
human operator, having as a goal to facilitate such an intuitive human/computer interaction within a mobile robot teleprogramming control scheme.

The main idea underlying this teleoperation scheme is related to a more fundamental pursuit of an efficient human-robot cooperation framework, where humans and robots take in charge the parts of the tasks that they can perform more efficiently. Many researches are currently concentrating their efforts in establishing such a "synergetic and collaborative" control framework, where the system combines in an "optimal" way the capacities of humans and robots, like for instance the "shared intelligence" scheme proposed in [2] for the planning of telemanipulation tasks. For the teleoperation of a mobile robotic platform, a simple application of this general principle could be to commit the human operator in performing the necessary global planning operations, which are more demanding in terms of complex reasoning and required "intelligence", while other more local tasks such as collision avoidance and trajectory optimization are dedicated to the telerobotic system. This section proposes an implementation of this principle, integrating a local path-planning method within the multimodal teleoperation interface presented above. The method is based on wavefront expansion of a simple distance metric and the use of a trajectory modification technique similar to vector field approaches. This scheme, called "computer-assisted teleplanning by human demonstration", aims at providing active assitance (collision avoidance and guidance) to the human operator, enabling him to indicate in a natural way the desired global plan to be followed by a remote mobile robot.

\section{A. Local Path Planning}

The problem of path-planning, that is, generating collision-free paths under particular taskrelated constraints, has attracted considerable interest for many years [1]. The two extreme approaches are: (a) global planning, based on a concise representation of the connectivity between collision-free configurations of the robot's workspace (usually in the form of a "connectivity graph"), and (b) local planning, which consists of searching locally around the robot for collision-free configurations, based on some heuristic that usually takes the form of a potential field guiding the search along the flow of its gradient vector field.

More recently, some research efforts have concen-

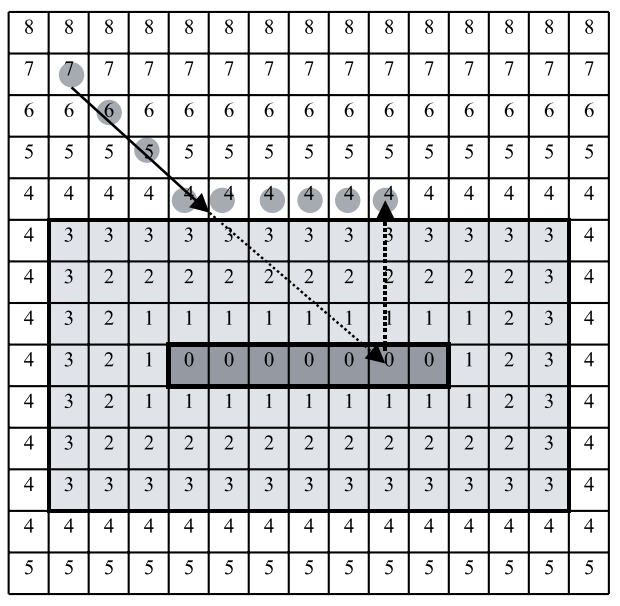

Fig. 3. Local path planning based on wavefront propagation of a distance metric

trated on integrating some form of motion planning technique within human/computer interaction systems. The goal is to develop "intelligent" interfaces that help users avoid unnecessary maneuvers, for instance avoid collisions when navigating within a virtual environment. In this context, a methodology was presented in [3] for supporting intelligent camera control in a $3 \mathrm{D}$ environment, incorporating a global path planning algorithm based on a roomto-room connectivity graph and global (static) numerical navigation function. Another method was proposed in [5], aiming also to facilitate user navigation within a virtual world, based on the integration of a probabilistic roadmap planner within a VRML interface for architectural walkthrough applications.

The drawback of using global path planning approaches is that they require an expensive precomputation step for the construction of the connectivity graph, while this step has to be repeated in case of a dynamically changing environment. Moreover, the graph search may also be time consuming, which may be inappropriate when real-time human-computer interaction applications are considered. We propose to integrate a simple local path planning method within a mobile robot teleoperation interface. This method is based on the automatic creation of a distance map based on a $2 \mathrm{D}$ top-view representation of the indoor environment constituting the robot workspace. This $2 \mathrm{D}$ map is constructed using a wavefront propagation algorithm for a standard Manhattan (L2) distance metric, considering a typical 8-connectivity for neigh- 


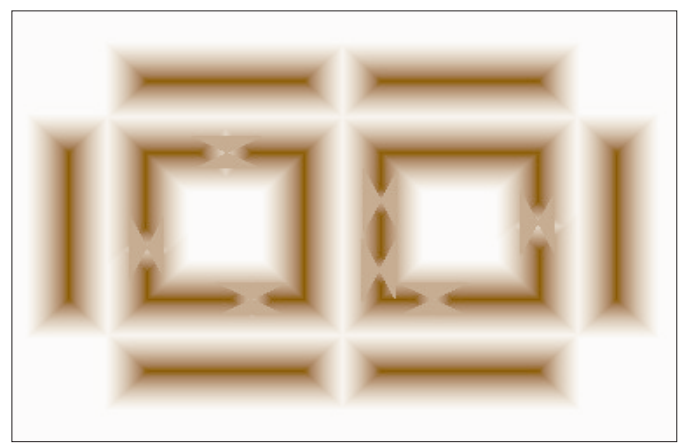

Fig. 4. Distance map for active collision avoidance

boring pixels. This is illustrated in Figure 3, where we see a commanded trajectory towards an obstacle (distance $=0$ ), and its modification following the gradient of the distance function until an acceptable threshold is reached (for the case of Figure 3, this threashold level equals 4).

This method intends to unburden the human operator from precisely and accurately inputing the desired trajectory to be followed. Instead, only an approximate global plan (room to room navigation) may be needed, while the system is in charge of dynamically correcting the input path, to deduce an appropriate trajectory for the mobile robot.

\section{B. Integration within the Teleoperation Interface}

The method has been implemented using Java, and integrated within the multimodal teleoperation interface presented in Section II. Fig. 4 shows a typical example of a distance map computed for an indoor environment (consisting of a couple of rooms, doorways and corridors). Fig. 5 demonstrates the implementation of this local path-planning method, for on-line anti-collision and guidance (following corridors, passing through doorways etc.). The arrows in this figure $(5-a, b, c)$ indicate the points where the algorithm intervenes to modify and correct the motion imparted by the human operator, and provide active assistance. Motion input can be provided by the human operator either using a standard mouse-drag procedure, or using other 2D devices like an e-pen digitizer. In the future we will consider human interaction using vision-based algorithms for natural gesture recognition.

An automatic waypoint generation module is then in charge of computing a set of optimal waypoints that will constitute the commanded robot motion plan (Figure 5-d). This can be performed

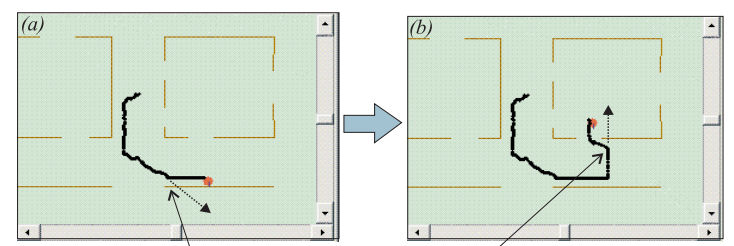

Active anti-collision and guidance

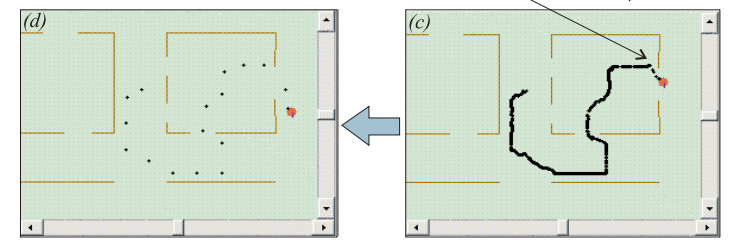

Automatic Waypoint Generation

Fig. 5. Computer-assisted trajectory teleplanning

either using some simple heuristic (like comparing simulated robot heading through neighboring points) or using some general optimization algorithm, taking into consideration constraints related to the motion control method employed by the mobile robotic platform. The system also supports previewing of the final planned trajectory (and even waypoint editing), in 2D and/or in 3D (VR) mode, in order to give to the human operator a realistic idea of the motion that is to be executed by the slave robot, prior to sending the deduced motion plan to the remote site for real execution (in the form of a list of waypoints with additional taskrelated information, such as visual landmark information etc.).

\section{Hardware Configuration of the Mobile Robotic Assistant}

Initial experiments are currently being performed using the multimodal VR-based teleoperation interface, with the local path-planning function active, for the remote control of an integrated mobile robotic assistant. The hardware configuration of the mobile robotic system consists of: (a) a mobile platform, manufactured by Robosoft and equipped with a ring of 16 ultrasonic sensors, (b) a vision system mounted on a pan/tilt platform, and (c) a small 5 dof manipulator arm, manufactured by Eshed Robotics, also integrated on the platform.

The robot platform is equipped with on-board computational power consisting of a VME-based controller (running on a Motorolla 68020 CPU processor), and a Pentium PC communicating via a wireless Ethernet link with the off-board central 

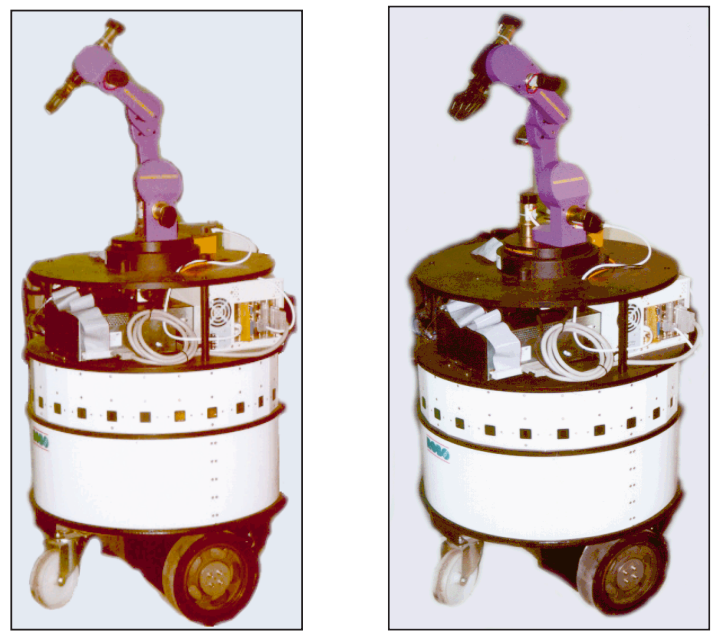

Fig. 6. Integrated mobile robotic assistant

control server. Figure 6 shows photos of the robotic system's current configuration, with the manipulator arm mounted on the platform. This work is performed in the context of a research project, called "HygioRobot"1, aiming at the development of an integrated mobile robotic system and the investigation of its use to perform assistive operations in a hospital environment [13][14] (for a survey on such systems, see [4], [6], [12]) The whole system should be fully operational for a complete series of experiments by the end of the year.

\section{Conclusions}

This paper focused on the design and implementation of a multimodal teleoperation interface, integrating VR models and Web standards, for the remote control of a mobile robotic assistant. We proposed to incorporate a local path planning technique within the user interface, based on a simple wavefront expansion algorithm of a standard distance metric. The method intends to facilitate global planning of the desired trajectory by the human operator, and enable intuitive user interaction and efficient operation of the system. Active computer-mediated assistance is provided to the human operator in the form of collision avoidance and active guidance, enabling him to indicate rapidly and in a natural manner the desired motion plan, without having to accurately input the precise mobile robot trajectory.

This mobile robot teleoperation scheme, called

${ }^{1}$ Participating partners are: National Techninal University of Athens, NCSR Demokritos and University of Pireaus computer-assisted teleplanning by human demonstration, is inspired by a more fundamental pursuit for an efficient human-robot collaboration framework, a general directive towards which many research efforts are now being oriented, intending to achieve a better synergy between humans and robots and exploit in an "optimal" way their capacities.

\section{REFERENCES}

[1] J. Barraquand, B. Langlois and J.-C. Latombe, "Numerical Potential Field Techniques for Robot Path Planning", IEEE Transactions on Systems, Man and Cybernetics, vol.22, no.2, pp.224-240, 1992.

[2] P. Bathia and M. Uchiyama, "A VR-Human Interface for Assisting Human Input in Path Planning for Telerobots", Presence, vol.8, no.3, pp.332-354, June 1999.

[3] S.M. Drucker and D. Zeltzer, "Intelligent Camera Control in a Virtual Environment", Graphics Interface'94, pp.190-199, 1994.

[4] J. E. Engelberger, "Health care robotics goes commercial - the helpmate experience", Robotica, VII: 517-523, 1993.

[5] T.-Y. Li and K.-K. Ting, "An Intelligent User Interface with Motion Planning for 3D Navigation", Proc. IEEE Virtual Reality Conference, pp. 177-184, 2000.

[6] E. Ettelt, R. Furtwängler, U. D. Hanebeck and G. Schmidt, "Design Issues of a Semi-Autonomous Robotic Assistant for Health Care Environment", Journal of Intelligent and Robotic Systems, vol.22, pp. 191-209, 1998.

[7] E. Freund and J. Rossmann, "Projective Virtual Reality: Bridging the Gap between Virtual Reality and Robotics", IEEE Transactions on Robotics and Automation, vol.15, no.3, pp.411-422, June 1999.

[8] K. Goldberg, "Introduction: The Unique Phenomenon of a Distance", in: The Robot in the Garden. Telerobotics and Telepistemology in the Age of the Internet, K. Goldberg (ed.), MIT Press 2000.

[9] A. Kheddar, C. Tzafestas, P. Coiffet, T. Kotoku, K. Tanie, "Multi-Robot Teleoperation Using Direct Human Hand Actions", International Journal of Advanced Robotics, Vol.11, No.8, pp.799-825, 1997.

[10] T. B. Sheridan, Telerobotics, Automation and Human Supervisory Control, The MIT Press, 1992.

[11] K Taylor and B. Dalton, "Internet Robots: A New Robotics Niche", IEEE Robotics and Automation Magazine, vol.7, no.1, (special issue: Robots on the Web), March 2000.

[12] S. G. Tzafestas, Guest Editorial, Journal of Intelligent and Robotic Systems: Special Issue on Autonomous Mobile Robots in Health Care Services, vol.22, nos.3-4, pp.177-179, July-August 1998.

[13] C. S. Tzafestas and D. Valatsos, "VR-based Teleoperation of a Mobile Robotic Assistant: Progress Report", Technical Report DEMO 2000/13, Institute of Informatics \& Telecom., NCSR Demokritos, November 2000.

[14] C. S. Tzafestas, "Multimodal Teleoperation Interface integrating VR Models for a Mobile Robotic Assistant", Proc. $10^{\text {th }}$ Intl. Workshop on Robotics in Alpe-AdriaDanube Region (RAAD'2001), Vienna, May 16-18, 2001.

[15] J. Vertut and P. Coiffet, Les Robots: Téléopération. Tome 3A: Evolution des technologies. Tome 3B: Téléopération assistée par ordinateur, Edition Hermes, Paris, 1984. 